\title{
AVANCES REALIZADOS EN EL ANÁLISIS DE MUESTRAS ÓSEAS RECUPERADAS DEL SITIO BOCA DE LEGA (SANTA FE, ARGENTINA) A PARTIR DE ESTUDIOS ARQUEOMÉTRICOS
}

\author{
Melania Lucila Lambri ${ }^{1}$, Ana María Rocchietti ${ }^{2}$, Mónica P. Valentini ${ }^{3}$, \\ Federico G. Bonifacih ${ }^{4}$, Patricia Bozzano ${ }^{5}$, Bernd Weidenfeller ${ }^{6}$, \\ Griselda I. Zelada ${ }^{7}$, Nélida De Grandis ${ }^{8}$ y Osvaldo A. Lambri ${ }^{9} 2$
}

Recibido: 29 de noviembre de 2018. Aceptado: 5 de abril de 2019

\begin{abstract}
Resumen
Se realizaron estudios arqueométricos que involucraron la aplicación de microscopía electrónica de barrido y técnicas termoanalíticas sobre distintas piezas óseas recuperadas del sitio de Boca de Lega (BL), Provincia de Santa Fe, Argentina. El material ósteoarqueológico recuperado de BL, exhibe una baja integridad mecánica y una distribución a nivel superficial, debido a procesos de acumulación de depósitos sedimentarios, re-depósitos y erosión del ambiente fluvial. También se observó signos de posible termoalteración. Los primeros estudios arqueométricos sobre un elemento denominado como BL21, lograron determinar la existencia de accionar antrópico de sociedades pretéritas. A partir de estos resultados, se avanzó en el análisis de otros elementos óseos de BL, denominados BL07, BL26 y BL49, mediante nuevos estudios arqueométricos en donde se aplicaron técnicas analíticas como ser: análisis térmico diferencial, termogramimetría y microscopía electrónica de barrido con
\end{abstract}

1 Facultad de Humanidades y Artes, Universidad Nacional de Rosario, Entre Ríos 758, S2000CRN, Rosario, Argentina.

CONICET-UNR, Lab. de Materiales, Esc. de Ingeniería Eléctrica, Centro de Tecnología e Investigación Eléctrica, Fac. de Cs. Exactas, Ingeniería y Agrimensura, Avenida Pellegrini 250, S2000BTP, Rosario, Argentina. E-mail: melania_lambri@hotmail.com

2 Facultad de Cs. Humanas, Universidad Nacional De Río Cuarto, Ruta Nacional 36 km 601, S5800, Río Cuarto, Argentina. E-mail: anaau2002@yahoo.com.ar

${ }^{3}$ Facultad de Humanidades y Artes, Universidad Nacional de Rosario, Entre Ríos 758, S2000CRN, Rosario, Argentina. E-mail: mopavalentini@gmail.com

4 CONICET-UNR, Lab. de Materiales, Esc. de Ingeniería Eléctrica, Centro de Tecnología e Investigación Eléctrica, Fac. de Cs. Exactas, Ingeniería y Agrimensura, Avenida Pellegrini 250, S2000BTP, Rosario, Argentina. E-mail: bonifaci@fceia.unr.edu.ar

5 Lab. de Microscopía Electrónica, Unidad de Actividad Materiales, Centro Atómico Constituyentes, CNEA, Instituto Sábato, Universidad Nacional de San Martín, Avenida General Paz 1400, B1650, San Martín, Buenos Aires, Argentina. E-mail: pbozzano@cnea.gov.ar

6 IEC, Department of Materials Science, Clausthal University of Technology, Arnold-Sommerfeld 6, 38678, Clausthal-Zellerfeld, Alemania.E-mail: bernd.weidenfeller@tu-clausthal.de

7 CONICET-UNR, Lab. de Materiales, Esc. de Ingeniería Eléctrica, Centro de Tecnología e Investigación Eléctrica, Fac. de Cs. Exactas, Ingeniería y Agrimensura, Avenida Pellegrini 250, S2000BTP, Rosario, Argentina. E-mail: gizelada@fceia.unr.edu.ar

8 Facultad de Humanidades y Artes, Universidad Nacional de Rosario, Entre Ríos 758, S2000CRN, Rosario, Argentina. E-mail: nellyisis@hotmail.com

9 CONICET-UNR, Lab. de Materiales, Esc. de Ingeniería Eléctrica, Centro de Tecnología e Investigación Eléctrica, Fac. de Cs. Exactas, Ingeniería y Agrimensura, Avenida Pellegrini 250, S2000BTP, Rosario, Argentina. E-mail: olambri@fceia.unr.edu.ar 
microanálisis dispersivo de energías. El uso conjunto de estas técnicas permitió identificar la alta incidencia de componentes edáficos en las muestras como también el registro de un calentamiento que fue superior a $673 \mathrm{~K} / 400^{\circ} \mathrm{C}$. En virtud de los resultados obtenidos fue posible reconfirmar el muy posible accionar humano como factor originario de la termoalteración previamente observada.

Palabras Clave: estudios arqueométricos, muestras óseas, técnicas termoanalíticas, SEM/ EDS, sitios fluviales.

\begin{abstract}
Archaeometric studies were carried out involving the application of thermoanalytical techniques and scanning electron microscopy on different bone pieces recovered from the site of Boca de Lega (BL), Province of Santa Fe, Argentina. The osteoarchaeological material recovered from BL exhibits a low mechanical integrity and a superficial morphology promoted from the accumulation of sedimentary deposits, re-deposits and the erosion of fluvial environment. There were also signals of possible thermo-alteration. The first archaeometric studies on the so called BL21 element, allowed us to determine the existence of anthropic actions of past societies. Continuing with these studies in a deeper mode, nowadays, progress was made in the analysis of other bone elements from BL, called BL07, BL26 and BL49, through new archaeometric studies. These involved analytical techniques such as differential thermal analysis, thermogrammetry and scanning electron microscopy with dispersive energy microanalysis. The joint use of these experimental techniques allowed us to identify the high incidence of edaphic components in the samples as well as the record of a heating that was superior to $673 \mathrm{~K} / 400^{\circ} \mathrm{C}$. By virtue of the results obtained, it was possible to reconfirm the human action as the highly probable original factor of previously observed thermo-alteration. Key Words: archaeometric studies, bone samples, thermoanalytical techniques, SEM/EDS, river sites.
\end{abstract}

\section{Introducción}

Los primeros estudios arqueométricos sobre material óseo del Noreste Argentino, en el Sitio Boca de Lega, Santa Fe, se realizaron correlacionaron resultados de espectroscopía mecánica, análisis térmico diferencial (DTA), calorimetría diferencial de barrido, termogravimetría (TGA), microscopía de luz, microscopía de luz computarizada con autofoco y análisis de imagen, microscopía electrónica de barrido (SEM) con análisis dispersivo de energía (EDS), espectroscopía de absorción infrarroja (FTIR), difracción de rayos $\mathrm{X} y$ fluorescencia de rayos $\mathrm{X}$; con el objetivo de determinar la naturaleza de los agentes transformadores, naturales o antrópicos, que pudieron afectarlo (Lambri 2018; Lambri et al. 2018a). Se ha podido distinguir la temperatura a la cual fue sometida la pieza arqueológica a través del estudio de la transición de la triple hélice colagénica al estado desordenado, TH $\rightarrow$ RC. Piezas calentadas a temperaturas inferiores a $\operatorname{los} 573 \mathrm{~K} / 300^{\circ} \mathrm{C}$, manifestaron una reacción endotérmica entre los $450 \mathrm{~K} / 177^{\circ} \mathrm{C}$ y $540 \mathrm{~K} / 267^{\circ} \mathrm{C}$, relacionada con el deterioro de la triple hélice colagénica; que involucra la transición TH $\rightarrow$ RC (Lambri 2018a; Lambri et al. 2014; Lambri et al. 2018; Mitche11 et al. 2005). En efecto, los procesos de deterioro en la estructura de las cadenas polipeptídicas que conforman las hélices colagénicas son termodinámicamente irreversibles, es decir, una vez que son destruidos, mayormente, desaparecen y no pueden volver a reconstruirse. Por lo tanto, al haberse medido la transición $\mathrm{TH} \rightarrow \mathrm{RC}$, esto indica que el material óseo no fue calentado por encima de alrededor de los $510 \mathrm{~K} / 237^{\circ} \mathrm{C}$. En contraste, los elementos 
que fueron calentados por encima de los $510 \mathrm{~K} / 237^{\circ} \mathrm{C}$, no presentaron la transformación $\mathrm{TH} \rightarrow \mathrm{RC}$. Consecuentemente, esto permitió determinar la posible existencia de accionar antrópico de sociedades pretéritas (Lambri 2018; Lambri et al. 2018a).

Cabe ser mencionado aquí que existen una importante cantidad de estudios arqueométricos focalizados a la diagénesis del material óseo por efecto de la temperatura, entre otros factores. En efecto, se pueden citar los trabajos de Shahack et at. (1997), Lebon et al. (2008), Cai y Singh (2004), Dal Sasso et al. (2016) utilizando principalmente FTIR, para el estudio de los cambios a nivel del colágeno y de la apatita fundamentalmente a partir de los cambios del así llamado splitting factor (IRSF (Artoli 2010) en su recristalización. Empleando las técnicas de SEM y TEM se pueden encontrar los trabajos de Hanson y Cain. (2007) y Nicholson (1993) entre otros, en donde se revelan aspectos morfológicos producidos por el deterioro térmico. Se han realizado también estudios empelando dispersión de rayos-X a bajos ángulos (SAXS) para determinar cambios en la morfología de la parte mineral. Además se han realizado estudios de TEM a nivel del colágeno (Koon et al. 2003) y también con microscopía de fuerzas atómicas para determinar los cambios en las fibrillas de colágeno en función de la temperatura (Bozec y Odlyha 2001).

De los trabajos arriba citados relacionados con el estudio del deterioro a temperaturas moderadas, por ejemplo una cocción, los estudios que pueden resultar más adecuados serían aquellos focalizados a determinar los cambios en la estructura colagénica, dada la estabilidad de la bioapaptita hasta temperaturas de alrededor de los $900 \mathrm{~K}$-1000 K (Artoli 2010; Kourkoumelis et al. 2012; Ooi et al. 2007).

En el presente trabajo se presentan estudios de DTA, TGA y SEM con EDS sobre nuevas piezas óseas recuperadas del sitio de Boca de Lega, con la intención de reportar avances en el estudio del material óseo de este sitio desde una perspectiva arqueométrica. En particular los estudios de SEM y EDS contemplaron tanto la exploración de las superficies interiores de las piezas obtenidas por cortes en secciones transversales como así también exploraciones de las superficies de las muestras en estado como recibido (sin lavar). El mapeo de EDS en este segundo caso, contempló todos los distintos elementos componentes del suelo correspondientes a la zona del sitio Boca de Lega. Esta comparación tiene por objeto evaluar el grado de transporte de materia desde la superficie al interior del hueso teniendo en cuenta el contexto fluvial del sitio.

\section{Descripción del Sitio}

El sitio de Boca de Lega (BL) está localizado a los $32^{\circ} 17^{\prime}$ '33.8' latitud sur y $60^{\circ}$ 52'25.7' longitud oeste, se encuentra catalogado, en función de la red ecológica, como albardón costero en isla, y se ubica en el sector inferior del río Paraná, entre las localidades Boca del Monje y Gaboto del departamento de San Jerónimo, Figura 1 (Rocchietti et al. 1997; Rocchietti et al. 2005). El mismo se encuentra en una zona islera con una topografia descrita por una colina costera dentro de una región inundable, formando parte, junto al sitio histórico colonial San Bartolomé de los Chaná (siglo XVII) y otros 30 sitios circundantes, del Polígono Gaboto-Monje (Polígono Gaboto Monje Ministerio de Innovación y Cultura 739/10 Dic. 2013), el cual está bajo estudio del equipo de Rocchietti y colaboradores desde los años 1997/98 (Rocchietti et al. 1997; Rocchietti et al. 2005). Concretamente, en el caso del sitio Boca de Lega, se observa un paisaje de bosques y pastizales (Rocchietti y De Grandis 2016; Rocchietti et al. 2005; Rocchietti et al. 2009). A su vez, este sitio presenta una distribución 


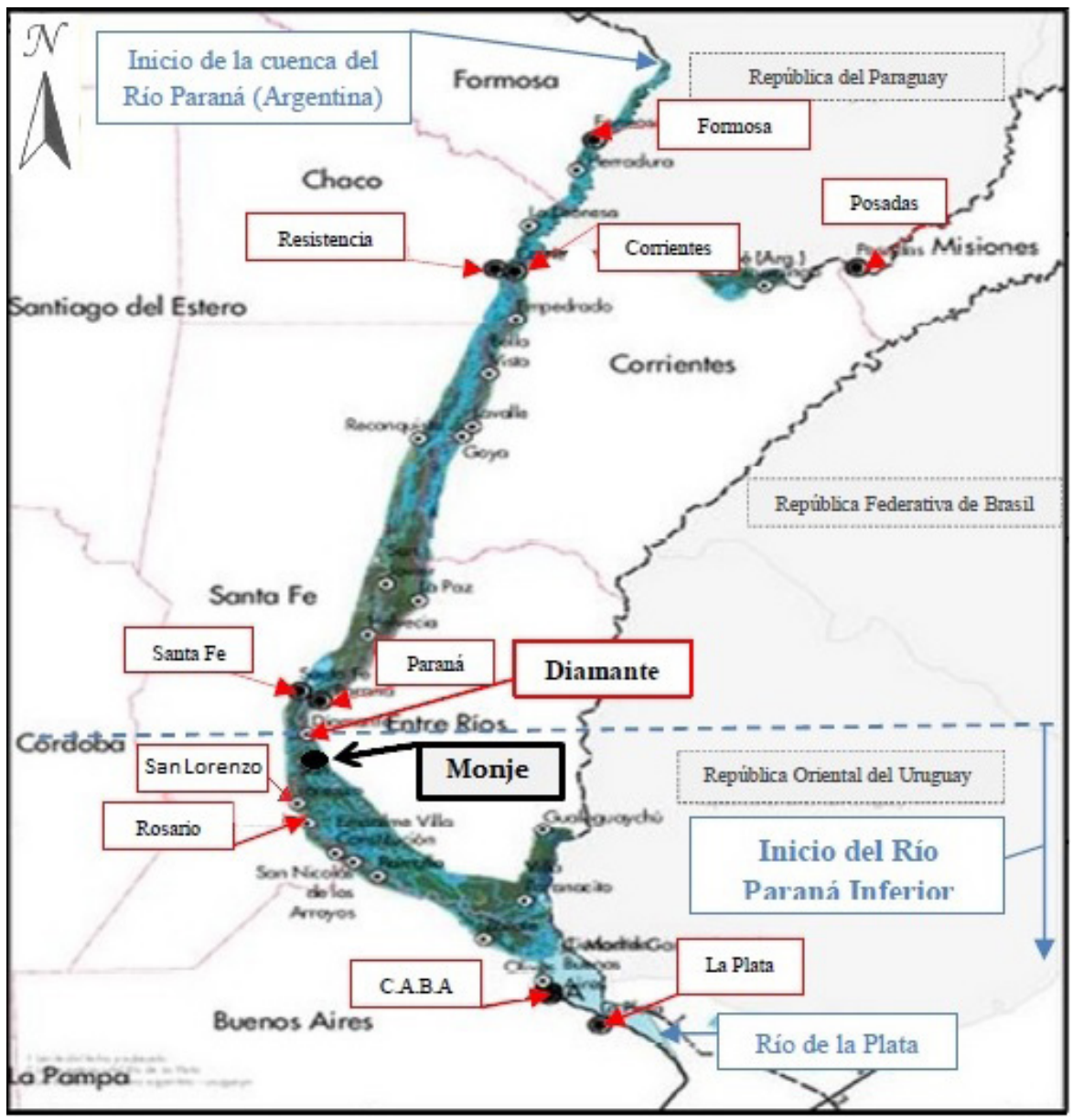

Figura 1. Ubicación de la localidad de Monje dentro de la Ecorregión Delta e Islas del Paraná. La linea punteada que atraviesa la localidad de Diamante constituye el punto de inicio de tramo Inferior del Paraná. Las zonas en azul corresponden a los cursos de agua. Las zonas verdes corresponden a los sectores de terreno. Los nombres de algunas ciudades relevantes para la orientación del lector se indican mediante flechas. Tomada y Modificado de Observatorio Nacional de Biodiversidad (2017).

del registro arqueológico a nivel superficial y una unidad edoestratigráfica húmica en el coronamiento del perfil, aunque también en algunos casos (como de inundación reciente) se encuentra por debajo de lentes arenolimosas o areno arcillosas (Rocchietti et al. 2005). De este mismo registro, fue posible recuperar una considerable cantidad de material cerámico, asociado a la cultura material Goya-Malabrigo, y en menor medida material óseo, vinculado a fauna, y leñoso. El material cerámico se 
encuentra bien estratificado y con una cota que los aísla durante los períodos de inundación. El material osteoarqueológico recuperado de $\mathrm{BL}$, exhibe una baja integridad mecánica, alto nivel de fragmentación y una distribución a nivel superficial debido a procesos de acumulación de depósitos sedimentarios, re-depósitos y erosión del ambiente fluvial. A su vez, también se observó signos de termoalteración. Para más información sobre el Sitio ver las referencias antes mencionadas en esta Sección.

\section{Equipos y procedimiento experimental}

\section{Muestras}

Las muestras estudiadas en este trabajo son secciones tomadas de los elementos denominados BL21, B126, BL49 y BL07, los cuales se presentan en la Figura 2 (Lambri 2018). Los elementos BL26 y BL49 comparten con BL21 una misma localización y nivel superficial de recolección, como también indicios de termoalteración y depositación sedimentológica. En BL07 se observan las mismas características que para las anteriores, pero con la diferencia de que éste fue recuperado en otra locación próxima y a nivel estratigráfico. Dichos elementos presentan, al igual que el resto del conjunto, un alto nivel de fragmentación que de momento ha impedido su identificación a una unidad o taxón específico.

\section{Mediciones}

Los estudios de las reacciones térmicas en función de la temperatura se realizaron mediante mediciones de DTA, las cuales se realizaron en un calorimetro diferencial convencional que permite ensayos desde $\operatorname{los} 233 \mathrm{~K} /-40^{\circ} \mathrm{C}$ hasta $1273 \mathrm{~K} / 1000^{\circ} \mathrm{C}$. Se utilizaron crisoles de acero inoxidable y las medidas se realizaron bajo atmósfera de argón a presión atmosférica. La velocidad de calentamiento utilizada en los ensayos fue controlada por un controlador de alta sensibilidad Lake Shore DRC-91C y fue de $5 \mathrm{~K} /$ minuto. La sensibilidad de resolución del sensor diferencial está en el orden del nanovoltio.

Las medidas de pérdida de peso en función de la temperatura se determinaron mediante ensayos de TGA realizados en una balanza electrodinámica de alta precisión, con una resolución de $0.1 \mathrm{mg}, \mathrm{BOE}-$ CO-Alemania, modificada como termobalanza. La lanza del portamuestra desde la balanza al horno de alta temperatura es un alambre de súper aleación de base cromo no magnético y el portamuestra es de acero inoxidable. El rango de temperatura admisible es desde temperatura ambiente (RT) hasta los $1373 \mathrm{~K} / 1100^{\circ} \mathrm{C}$. Las medidas se realizaron en aire con una velocidad de calentamiento de $5 \mathrm{~K} /$ minuto, controlada por un controlador Novus-1000. El error en la variación de pérdida de peso fue menor al 1\%. El tamaño de las muestras para las técnicas antes mencionadas es de alrededor de $3 \mathrm{~mm} \times 3 \mathrm{~mm} \times 2 \mathrm{~mm}$.

Los relevamientos con microscopía óptica de luz (LM) se realizaron en un microscopio invertido metalográfico Arcano, con análisis digital de imagen con cámara de alta resolución con enfoque y magnificación adicional secundario, trabajando con haz rasante de luz blanca.

Los estudios de SEM en las secciones transversales de las muestras se realizaron en un microscopio de emisión de campo (FE) FE-SEM Jeol JSM 7610F, Japan, equipado con EDS a través de un dispositivo EDAX en el IEC, Department of Materials Science, Clausthal University of Technology, Clausthal-Zellerfeld, Germany. Mientras que las exploraciones de las superficies en las muestras como recibidas se realizaron en un microscopio electrónico FEI Quanta 200 operado a $15 \mathrm{kV}$ bajo vacío, en el Laboratorio de Microscopía 

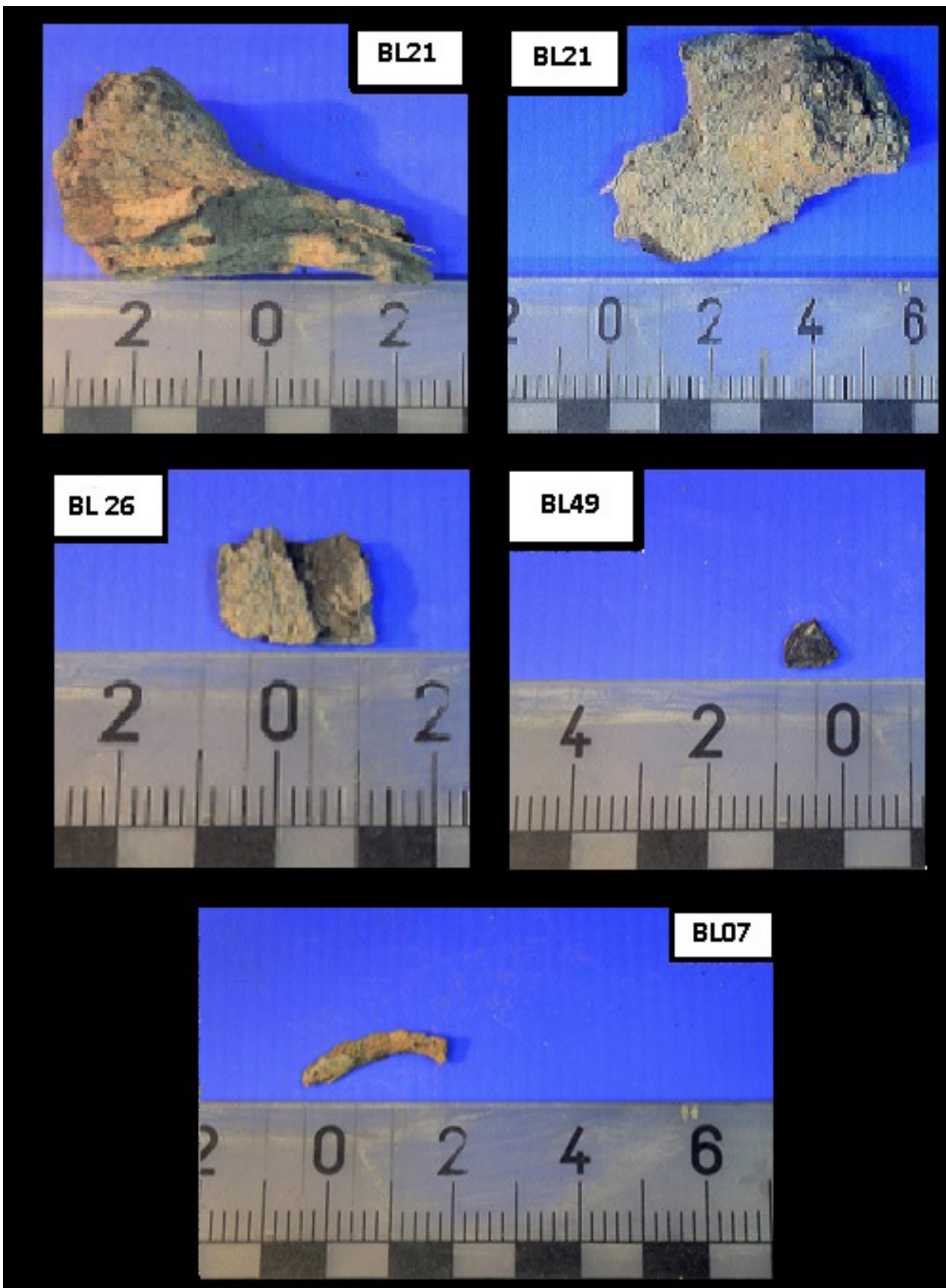

Figura 2. Elementos BL021 vista lateral (Izquierda Arriba) y vista frontal (Derecha Arriba), BL026 (Izquierda Centro), BL49 (Derecha Centro) y BL07 (Abajo Centro). Muestras en estado como recibido. 
Electrónica de la Unidad de Actividad Materiales del Centro Atómico Constituyentes de la Comisión Nacional de Energía Atómica, Argentina.

\section{Resultados}

El termograma medido para la muestra BL21 se presenta en el eje izquierdo de la Figura 3, mediante la curva llena. Este exhibe reacciones endotérmicas en intervalos de temperaturas entre $300 \mathrm{~K}-400 \mathrm{~K} / 27$ ${ }^{\circ} \mathrm{C}-127^{\circ} \mathrm{C}, 490 \mathrm{~K}-550 \mathrm{~K} / 217^{\circ} \mathrm{C}-277$ ${ }^{\circ} \mathrm{C}, 750 \mathrm{~K}-850 \mathrm{~K} / 477^{\circ} \mathrm{C}-577^{\circ} \mathrm{C}$; y reacciones exotérmicas en intervalos $400 \mathrm{~K}$ $490 \mathrm{~K} / 127^{\circ} \mathrm{C}-217^{\circ} \mathrm{C}, 600 \mathrm{~K}-700 \mathrm{~K} / 327$ ${ }^{\circ} \mathrm{C}-427{ }^{\circ} \mathrm{C}$ y desde $900 \mathrm{~K} / 627^{\circ} \mathrm{C}$ hasta alrededor de $1000 \mathrm{~K}-727^{\circ} \mathrm{C}$ (Lambri 2018; Lambri et al. 2018a; Lambri et al. 2018b). La secuencia de reacciones endotérmicas por debajo de los $400 \mathrm{~K}\left(127^{\circ} \mathrm{C}\right)$ seguidas de reacciones exotérmicas a temperaturas mayores a los $450 \mathrm{~K} / 177^{\circ} \mathrm{C}$ corresponden a las arcillas de granulometría fina y media, los suelos limosos (Leinweber et al. 1992; Smykatz-Kloss 1974) y también el humus (Shurygina et al. 1971). Asimismo, la reacción endotérmica a alrededor de los
$850 \mathrm{~K} / 577{ }^{\circ} \mathrm{C}$ también se asocia a suelos arenosos (Leinweber et al. 1992; SmykatzKloss 1974). En particular cabe destacarse que el termograma para BL2 1 exhibe el pico correspondiente a la $\mathrm{TH} \rightarrow \mathrm{RC}$ a alrededor de los 510K (Lambri 2018; Lambri et al. 2018a; Lambri et al. 2018b). Todas estas reacciones observadas mediante DTA se corresponden a su vez con las caídas de peso registradas en TGA, ver eje derecho en la Figura 3, las cuales están asociadas con el deterioro térmico de los diversos componentes edáficos identificados.

La respuesta térmica para la muestra BL007 es similar a la registrada para la muestra BL21.

Para las muestras BL26 (linea de trazos) y BL49 (línea de trazos alternados), las intensidades de las reacciones endotérmicas y exotérmicas hasta $800 \mathrm{~K}$ son más fuertes (ver eje izquierdo en la Figura 3). Sin embargo, debe destacarse que en las muestras BL26 y BL 49 no se pudo apreciar la aparición del pico relacionado con la transición TH $\rightarrow$ RC (Lambri et al. 2018b).

Las pérdidas de peso en función de la temperatura para BL26 y BL49 (ver el eje izquierdo en Figura 3 y símbolos) son
Figura 3. Termogramas de DTA (eje izquierdo y líneas) y TGA (eje derecho y simbolos) de los elementos BL21 (linea llena y círculos), BL26 (linea de trazos y triángulos) y BL 49 (línea de trazos alternada y triángulos invertidos).

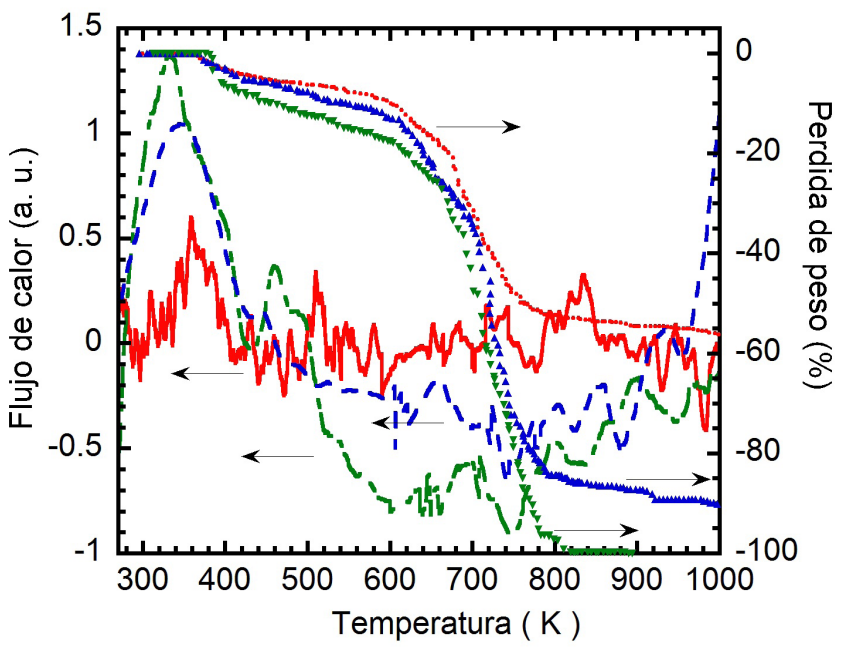


también mayores que para las muestras BL21 y BL07. En efecto, para BL26y BL49 se pueden observar en la curva de TGA la primera caída a alrededor de los 400K/ 127 ${ }^{\circ} \mathrm{C}$ y una marcada caída a alrededor de los $600 \mathrm{~K} / 327^{\circ} \mathrm{C}$ mayor al 80\%. Sin embargo, la pequeña caída a alrededor de los $510 \mathrm{~K}$ relacionada con la transición del colágeno no pudo ser detectada; en acuerdo con el termograma de DTA para estas muestras.

Las observaciones de SEM con EDS para las muestras de secciones transversales de los elementos BL21, BL07 y BL26 se presentan en la Figura 4. Como se mencionó en la Sección anterior, las micrografias mostradas y sus EDS corresponden a una zona tomada para cada uno de los elementos arqueológicos. La morfología para la muestra BL49 y su EDS resultaron similares a los correspondientes de BL26. Como puede observarse de las micrografias, las cavidades en BL26 son mayores que para BL21 y BL07 permitiendo una mayor acumulación de sedimentos. Las cavidades exhiben mayormente forma alargada con ejes con valores medios entre los $50-100 \mu \mathrm{m}$, $10-20 \mu \mathrm{m}$ y $100-200 \mu \mathrm{m}$, para las muestras BL21, BL07 y BL26; respectivamente, en acuerdo con huesos que han estado sumergidos en agua (Arenas Alatorre et al. 2007; López et al. 2011; Trujillo-Mederos et al. 2012).

El mayor tamaño de las cavidades para las muestras BL26 y BL49 puede estar relacionado con un mayor grado de calentamiento, el cual lleva a un mayor grado de deterioro de la hélice colagénica (Lambri et al. 2018b). Teniendo en cuenta la morfología de las muestras BL26 y BL49 y la locación de las mismas en terrenos inundables, la mayor cantidad de cavidades internas óseas, y a su vez mayores en tamaño, que en BL21y BL07, permiten recolectar mayor cantidad suelo y por lo tanto son mayores las reacciones endotérmicas y exotérmicas relacionadas con los suelos arcillosos, limosos, húmicos; en acuerdo con las me- diciones de DTA y TGA en la Figura 3. Asimismo, los resultados de EDS confirman los elementos de los componentes edáficos correspondientes al Sitio (Lambri 2018), determinados desde DTA y TGA.

De los diferentes estudios de EDS realizados a las diferentes secciones de las muestras arqueológicas en este trabajo también se han encontrado $\mathrm{P}, \mathrm{S}, \mathrm{Mg}, \mathrm{F}$ y $\mathrm{K}$; estos tres últimos asociados a los elementos edáficos (Lambri 2018).

Cabe también mencionarse que desde la vasta exploración de EDS fue posible determinar en todas las muestras estudiadas la ausencia de depósitos de Manganeso

Las diferencias de los EDS para las diferentes secciones de las muestras arqueológicas en relación con los componentes edáficos tienen que ver con las características dinámicas donde se encuentra emplazado el sitio. La detección aleatoria del $\mathrm{P}$ tiene que ver con el enmascaramiento de este elemento en frente a las diferentes proporciones de depositaciones, con la menor concentración que el Ca y con la proximidad de las energías de absorción entre el $\mathrm{P}$ y el $\mathrm{S}$.

Las observaciones de SEM y los mapeos de EDS a las superficies de las muestras (no secciones transversales) como recibidas se presentan en la Figura 5 para la muestra BL07, de donde se puede observar el acuerdo de los elementos de los componentes edáficos obtenidos desde las exploraciones de las secciones transversales. Asimismo, la distribución de los componentes resulta bastante homogénea en la superficie, a pesar obviamente de zonas con mayores concentraciones. Además para las muestras BL21, BL 26 y BL 49 se obtuvieron mapeos con similares características. Por lo tanto, el transporte de materia desde el suelo al interior de las cavidades ha existido, en acuerdo con la locación del material osteoarqueológico en sitios inundables de alta energía hídrica. 


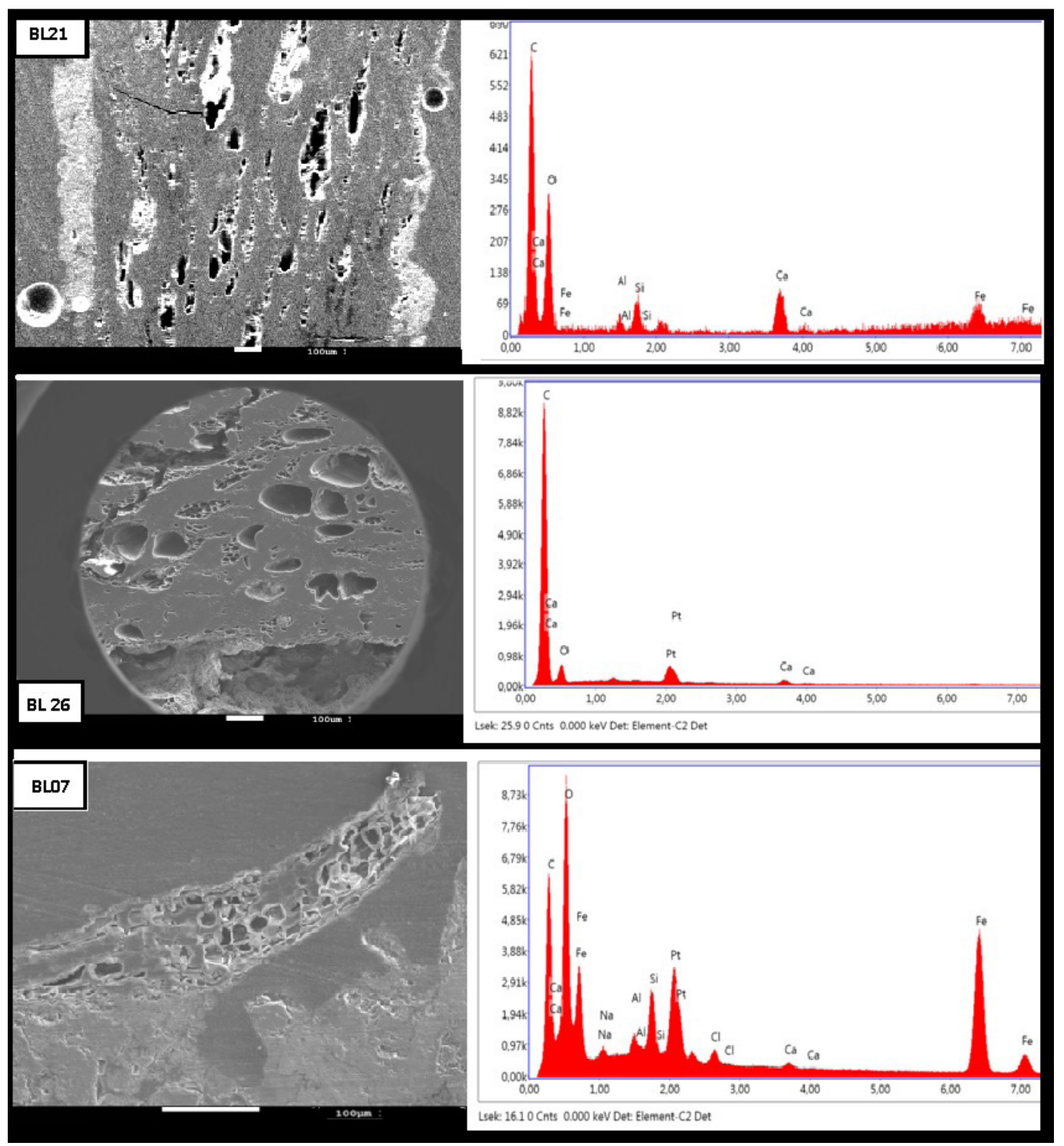

Figura 4. Micrografias de SEM y espectros de EDS para las muestra BL21, BL26 y BL07.

\section{Consideraciones finales}

Los resultados desde las técnicas termoanaliticas de DTA y TGA revelan diferencias entre las muestras BL21 y BL07 por un lado y BL26 y BL49 por el otro, no solo en relación a la cantidad del contenido de depositaciones sino también en cuanto a la presencia a alrededor de los $510 \mathrm{~K} / 237^{\circ} \mathrm{C}$ de la reacción endotérmica relacionada con 


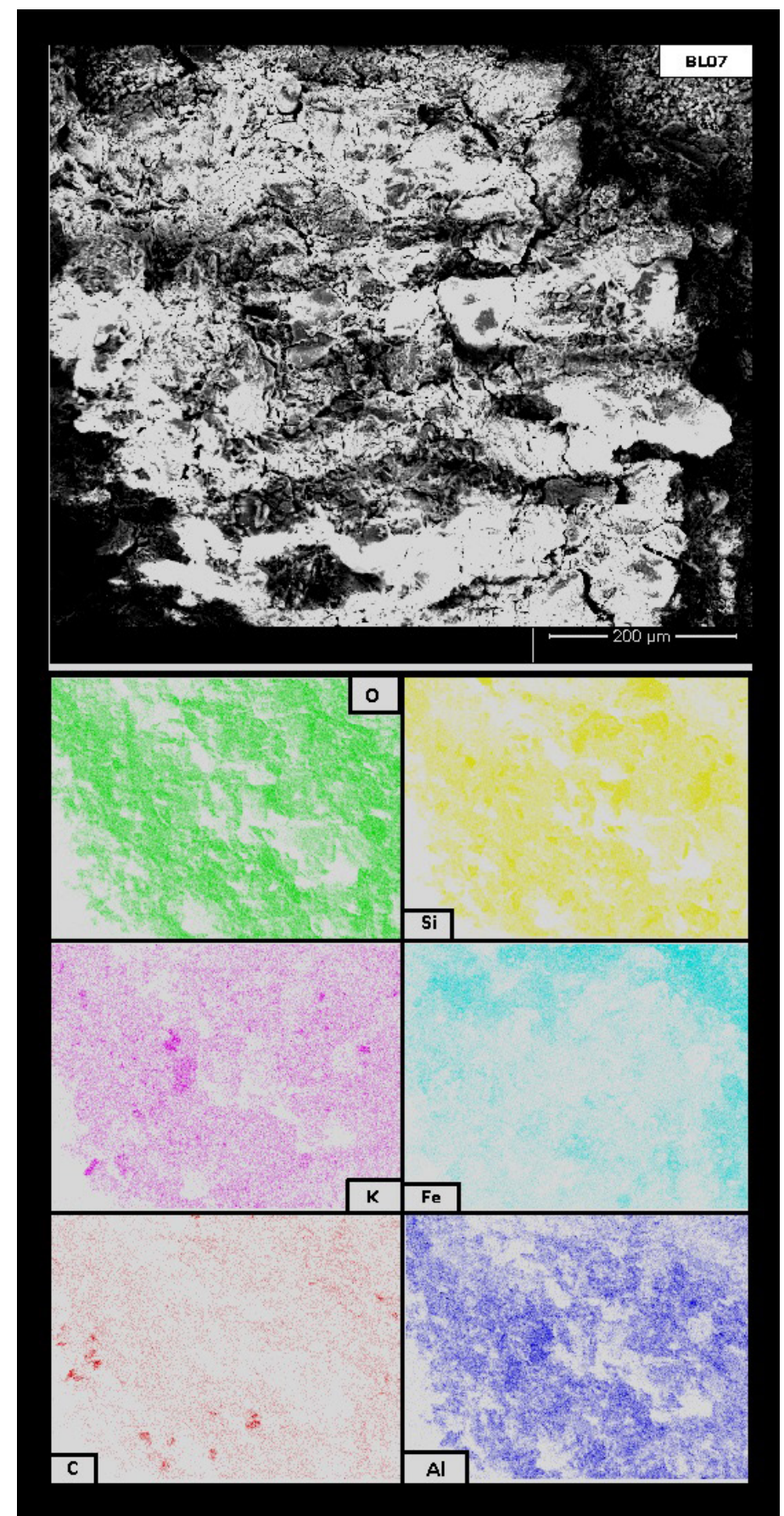

Figura 5. Mapeo de elementos (O, $\mathrm{K}, \mathrm{Fe}, \mathrm{C}, \mathrm{Al}, \mathrm{Si}$ ) para la superficie de una muestra BL07 como recibida. Arriba micrografia SEM para BL07. 
la transición de la triple hélice colagénica hacia el estado desordenado $(\mathrm{TH} \rightarrow \mathrm{RC})$. En efecto, las muestras BL21 y BL107 presentan esta reacción por lo tanto, en acuerdo con lo comentado precedentemente estas muestras no pueden haber sido calentadas por encima de esta temperatura. En contraste, para las muestras BL26 y BL 49, la ausencia de esta reacción indica que las mismas fueron calentadas por encima de los $510 \mathrm{~K} / 237^{\circ} \mathrm{C}$. Además la morfología de las cavidades en las secciones transversales de las muestras, reveladas desde observaciones de SEM exhiben erosión en acuerdo con muestras que han estado en ambientes inundables con alta energía hídrica. Más aún, la aparición de los elementos edáficos en secciones transversales centrales al volumen del elemento recolectado, que tienen correspondencia con los elementos mapeados desde SEM y EDS en las superficies de los elementos arqueológicos, apuntarían también a un proceso de transporte de masa (elementos edáficos) en medios líquidos, más que un proceso de tipo difusión en sólidos.

En virtud de los resultados obtenidos, se resalta la potencialidad y necesidad del desarrollo de la arqueometría para el estudio de restos ósteoarqueológicos recuperados de sitios ubicados en contextos fluviales.

\section{Bibliografia}

ARENAS ALATORRE, J.A.; S. SÁNCHEZ PÉREZ; A. DEL ÁNGEL ESCALONA; O. STERPONE; C. ZORRILLA y A. GÓMEZ SERRANO. 2007. Diagénesis en huesos humanos de la época colonial del Estado de Hidalgo, México. En Estudios de Antropología Biológica Volumen XIII, editado por M. Civera Cerecedo y M.R. Herrera Bautista, pp. 361-580. Universidad Nacional Autónoma de México, Instituto de Investigaciones Antropoló- gicas, Instituto Nacional de Antropología e Historia y Asociación Mexicana de Antropología Biológica, México.

ARTOLI, G. 2010. Scientific methods and cultural heritage. An introduction to the application of materials science to archaeometry and conservation science. Oxford University Press, Oxford.

BOZEC, L. y M. ODLYHA. 2001. Thermal denaturation studies of collagen by microthermal analysis and atomic force microscopy. Biophysical Journal 101:228-236.

CAI, S. y B.R. SINGH. 2004. A Distinct Utility of the Amide III Infrared Band for Secondary Structure Estimation of Aqueous Protein Solutions Using Partial Least Squares Methods. Biochemistry 43:2541-2549.

DAL SASSO, G.; M. LEBON; I. ANGELINI; L. MARITAN; D. USAI y G. ARTIOLI. 2016. Bone diagenesis variability among multiple burial phases at Al Khiday (Sudan) investigated by ATR-FTIR spectroscopy. Palaeogeography, Palaeoclimatology, Palaeoecology 463:168-179.

HANSON, M. y C. CAIN. 2007. Examining histology to identify burned bone. Journal of Archaeological Science 34(11):1902-1913.

KOON, H.; R. NICHOLSON y M. COLLINS. 2003. A practical approach to the identification of low temperature heated bone using TEM. Journal of Archaeological Science 30:1393- 1399.

KOURKOUMELIS, N.; A. LANI y M. TZAPHLIDOU. 2012. Infrared spectroscopic assessment of the inflammation-mediated osteoporosis (IMO) model applied to rabbit bone. Journal of Biological Physics 38:623-635.

LAMBRI, M.L.; E.D. GIORDANO; P.B. BOZZANO; F.G. BONIFACICH; J.I. PÉREZ-LANDAZÁBAL; G.I. ZELADA; D. GARGICEVICH; V. RECARTE y O.A. LAMBRI. 2016. Thermal Degradation of Type I Collagen from Bones. Journal of 
Renewable Materials 4:251-257.

LAMBRI, M.L. 2018. Estudios Arqueométricos aplicados a elementos óseos de los sitios prehispánicos e históricos emplazados en islas y costa del río Paraná inferior (Santa Fe, Argentina). Tesis de Licenciatura, Escuela de Antropología, Facultad de Humanidads y Artes, Universidad Nacional de Rosario.

LAMBRI, M.L.; A.M. ROCCHIETTI; F.G. BONIFACICH; B. WEIDENFELLER; M. VALENTINI; G.I. ZELADA; N. DE GRANDIS y O. A. LAMBRI. 2018a. Archaeometric study on bones from the Boca de Lega Site, Santa Fe, Argentina. Actas VII Congreso Nacional de Arqueometria, pp. 54-57. Universidad Nacional de Tucumán, Tucumás.

LAMBRI, M.L.; A.M. ROCCHIETTI; F.G. BONIFACICH; B. WEIDENFELLER; P.B. BOZZANO; M. VALENTINI; G.I. ZELADA; N. DE GRANDIS y O. A. LAMBRI. 2018b. Experimental study for the determination of the degree of thermal degradation in bones. Its application to the study of archaeological bones remains from the Boca de Lega Site. Manuscrito a ser enviado a Journal of Archaeological Science.

LAMBRI, O.A.; J.I. PÉREZ-LANDAZÁBAL; F.G. BONIFACICH; V. RECARTE; M.L. LAMBRI; G.I. ZELADA; F. TARDITTI y D. GARGICEVICH. 2014. Damping Micromechanisms for Bones Above Room Temperature. Journal of Biomimetics, Biomaterials, and Tissue Engineering 19:87-98.

LEBON, M.; I. REICHE; F. FRÖHLICH; J.J. BAHAIN y C. FALGUĖRES. 2008. Characterization of archaeological burnt bones: contribution of a new analytical protocol based on derivative FTIR spectroscopy and curve fitting of the v1v3 PO4 domain. Analytical and Bioanalytical Chemistry 392:1479-1488.

LEINWEBER, P.; H.R. SCHULTEN y C. HORTE. 1992. Differential thermal analysis, thermogravimetry and pyrolysis-field ionisation mass spectrometry of soil organic matter in particle-size fractions and bulk soil samples. Thermochimica Acta 194:175-187.

LÓPEZ P.; I. CARTAJENA; G. OLIVARES; Ó. LÓPEZ; D. CARABIAS y C. MORALES. 2011. Aplicación de Microscopio Electrónico de Barrido (MEB) y Espectroscopia de Energía Dispersiva (EDS) para distinguir alteraciones térmicas en restos osteofaunísticos de un sitio sumergido del Pleistoceno Final de la costa de Chile Central. En Temas de Arqueología: Estudios Tafonómicos y Zooarqueológicos (Volumen II), editado por A. Acosta, D. Loponte y L. Mucciolo, pp 25-44. Instituto Nacional de Antropología y Pensamiento Latinoamericano, Buenos Aires.

MITCHELL, P.C.H.; S.F. PARKER; A.J. RAMÍREZ-CUESTA y J. TOMKINSON. 2005. Vibrational Spectroscopy with Neutrons With Applications in Chemistry, Biology, Materials Science and Catalysis. En Series on Neutron Techniques and Applications. Vol. 3, pp. 474. World Scientific Publishing, New Jersey.

NICHOLSON, R. 1993. A morphological investigation of burnt animal bone and an evaluation of its utility in archaeology. Journal of Archaeological Science 20:411-428.

OOI, C.Y.; M. HAMDI y S. RAMESH. 2007. Properties of hydroxyapatite produced by annealing of bovine bone. Ceramics International 33:1171-1177.

POLÍGONO GABOTO MONJE, MINISTERIO DE INNOVACIÓN Y CULTURA 739/10 Dic. 2013

ROCCHIETTI, A.M.; N. GRANDIS; V. VICIOSO; J. BARUSCIA y L. MARTÍNEZ. 1997. La boca del arroyo Monje: los indios isleros y la invasión europea en siglo XVII. Actas de las Jornadas de Antropología de la Cuenca del Plata 111, pp. 188-195. Escuela de Antro- 
Lambri et al. - "Avances realizados en el análisis de muestras óseas recuperadas ..."

pología, Universidad Nacional de Rosario, Rosario.

ROCCHIETTI, A.M.; N. DE GRANDIS y M. VALENTINI. 2005. Arqueología de costa e islas del Paraná santafesino: área Monje-Gaboto. Revista de la Escuela de Antropologia (UNR) 10:41-54.

ROCCHIETTI, A.M.; N. DE GRANDIS y M. VALENTINI. 2009. Arqueología y paisaje cultural-natural de los indios isleros de la Provincia de Santa Fe, Documento Digital: www.icomos.org.ar/wp-content/uploads/2009/08/04_rocchietti_et_al.pdf. Consultado: 01/08/2017.

ROCCHIETTI, A.M. y N. DE GRANDIS. 2016. Economia y sociedad en una reducción indígena en el litoral del Paraná. Revista de Arqueología Histórica Argentina y Latinoamericana 10(1):91-117.

SHAHACK-GROSS, R.; O. BAR-YOSEF y S. WEINER. 1997. Black-Coloured Bones in Hayonim Cave, Israel: Differentiating Between Burning and Oxide Staining. Journal of Archaeological Science 24:439-446.

SHURYGINA, E.A.; N.K. LARINA; M.A. CHUBAROVA y M.M. KONONOVA, M. M. 1971. Differential thermal analysis (DTA) and thermogravimetry (TG) of soil humus substances. Geoderma 6:169177.

SCHALLER, R.; S. BARRAULT y P.H. ZYSSET. 2004. Mechanical spectroscopy of bovine compact bone. Material Science Engineering 370:569-574.

SCHALLER, R.; G. FANTOZZI y G. GREMAUD (Eds.) 2001. Mechanical Spectroscopy. TransTech Publications, Suiza.

SMYKATZ-KLOSS, W. 1974. Differential Thermal Analysis: Application and Results in

Mineralogy. Springer, Nueva York.

TRUJILLO-MEDEROS, A.; I. ALEMÁN; M. BOTELLA y D.P. BOSCH. 2012. Changes in human bones boiled in seawa- ter. Journal of Archaeological Science 39:1072-1079. 
